\title{
Variations of Images to Increase Their Visibility
}

\begin{abstract}
Amelia Carolina Sparavigna ${ }^{1}$
${ }^{1}$ Department of Applied Science and Technology, Politecnico di Torino, Torino, Italy

Abstract: The calculus of variations applied to the image processing requires some numerical models able to perform the variations of images and to extremize an appropriate actions. To produce the variations of images, there are several possibilities based on the brightness maps. Before a numerical model, I propose an experimental approach, based on a tool of Gimp, the GNU Image Manipulation Program, in order to visualize how the image variations can be. After the discussion of this tool, which is able to strongly increase the visibility of images, the variations and a possible functional for the visibility are discussed in the framework of a numerical model. The visibility functional is analogous to the fringe visibility of the optical interference.
\end{abstract}

Keywords: Image visibility, Fringe visibility, GIMP, Image processing

\section{Introduction}

Sometimes, the processing of images is performed to have their optimization in some way. This is the same aim of the variational calculus. This calculus is dealing with the problem of finding the extremal values of functionals. As maps from a set of functions to real numbers, the functionals are often defined as definite integrals of functions and their derivatives. If we consider an image, it is possible to have several functions and functionals from it. For instance, to each pixel at the arbitrary point $\mathrm{P}(\mathrm{x}, \mathrm{y})$ in the image frame we can associate a grey or colour tone $b$ ranging from 0 to 255: $\mathrm{b}(\mathrm{x}, \mathrm{y})$ is then a 2-dimensional function representative of the image intensity (brightness) distribution. From the brightness function, we can have a histogram, giving the number of pixels having a specific tone b. Once the functions have been defined, it is possible to devise a functional and associate the calculus of variations to the image processing [1].

Before discussing the image processing, let us remember some well-known problems of the calculus of variation. One problem is that of finding the curve of shortest length connecting two points. In a flat space, the solution is a straight line between the points. If we are in a curved space, there is the possibly of different solutions, known as geodesics. Another example is the Fermat's principle, which tells that the light follows the path of shortest optical length connecting two points.

In mechanics, this principle is known as the principle of least action. The action is a functional having as argument the trajectory, also called the "path" of the system, and a real number as its result [2]. Generally, the action takes different values for different paths. Classical mechanics postulates that the path really followed by a physical object is that minimizing the action (Figure 1 is adapting an image of the Feynman's book [2]). The equations of motion of a physical system are derived from the principle of least action, by means of the variational calculus. Each path, $x(t)$, from the initial to the final position has its action. We change the path of a small amount, the variation $\eta(t)$. The new path has a different value of its action, but it is that having the lowest action, that becomes the true trajectory of the body.

In image processing, the calculus of variations is used for the image reconstruction, for instance denoising and deblurring, and for segmentations [1]. The calculus then requires the numerical solution of models able to create the variations of images and then the minimization of some appropriate actions. To produce the variations of images, there are several possibilities based on the brightness map of the image. Before a numerical calculus, here we show an experimental approach, based on a tool of Gimp, GNU Image Manipulation Program [3], in order to visualize the image variations. After the discussion of this tool, which is able to increase strongly the visibility of images, the variations and a possible functional for the visibility are discussed with a numerical model. 


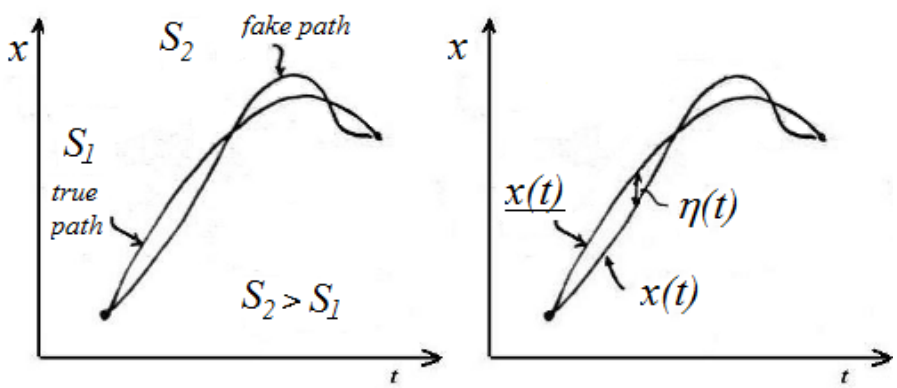

Fig.1 This image, adapted from the "Lectures on Physics" by Feynman et al., illustrates the variational calculus in mechanics. To find the path, truly followed by the system, we have to calculate the action for several variations of the path. The true path is that minimizing the action $S$. The variation of the path is given by the function $\eta(t)$.

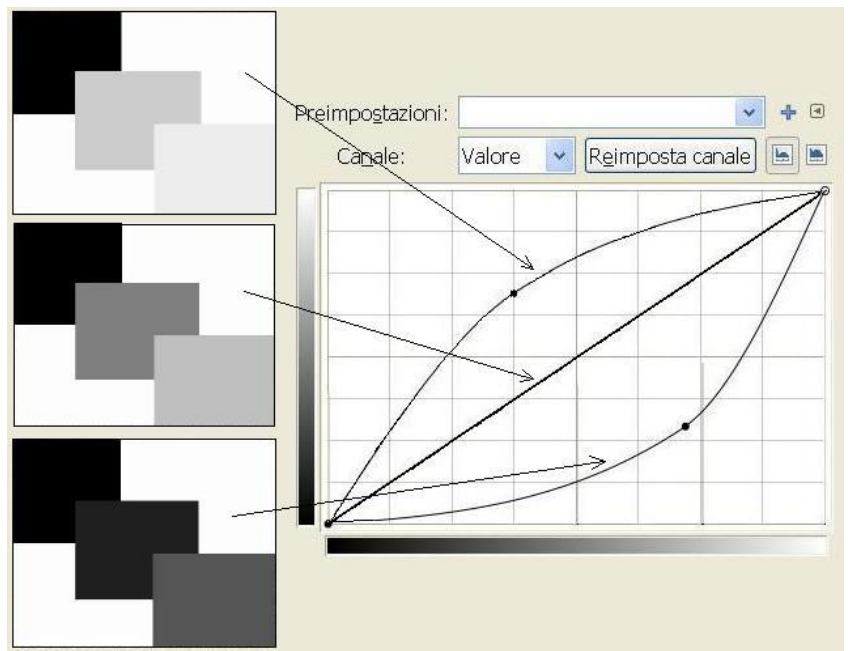

Fig.2: The "Curves" tool of Gimp changes the image. Dragging the curve above the diagonal, which correspond to the original image, the intensity is increased; dragging below, the intensity is decreased. The original image has only four tones: black, white and two greys. Note the variation of the image on the left as a consequence of the variation of the curve, shown on the right. These curves looks like the graphs in the Figure 1. Note that the tones black and white remain at their fixed values as the initial and final points of the Figure 1.

\section{The "Curves" tool of Gimp}

Let us consider the simple case where the variation of the image is obtained by changing its brightness (more complex variations could be obtained changing shape and position of the objects in the image frame, but this is beyond the aim of this paper). To visualize how the variation can be implemented, let us use the "Curves" tool of the Gimp. "Curves" is used to control brightness, contrast and colour balance quite freely. To open this tool, the path is the following: <Image> Tools $>$ Colour Tools $>$ Curves. A window such as that in the right part of the Figure 2 appears. As we open Curves, we see a panel reporting the histogram of the image and a straight line. Note that $\mathrm{x}$ - and $\mathrm{y}$ - axes have the grey tones as scales. The curve defines how to change the intensity of the image, with respect to the diagonal line, which is representing the image as it is. Dragging the curve above the diagonal, the intensity is increased; dragging below, the intensity is decreased. It is then possible to adjust the channel "Value", which will brighten or darken the image, moving the line up or down. Moreover, it is also possible to adjust the Red, Blue, Green, and Alpha channels, separately. The curve is able to redistribute contrast, because a steeper curve gives more contrast, whereas a flat curve produces a dull image. Figure 2 shows an example of the curve manipulation. The original image has only four tones: black, white and two greys. Note the variation of the image on the left as a consequence of the variation of the curve, shown on the right. These curves looks like the paths in the Figure 1. 


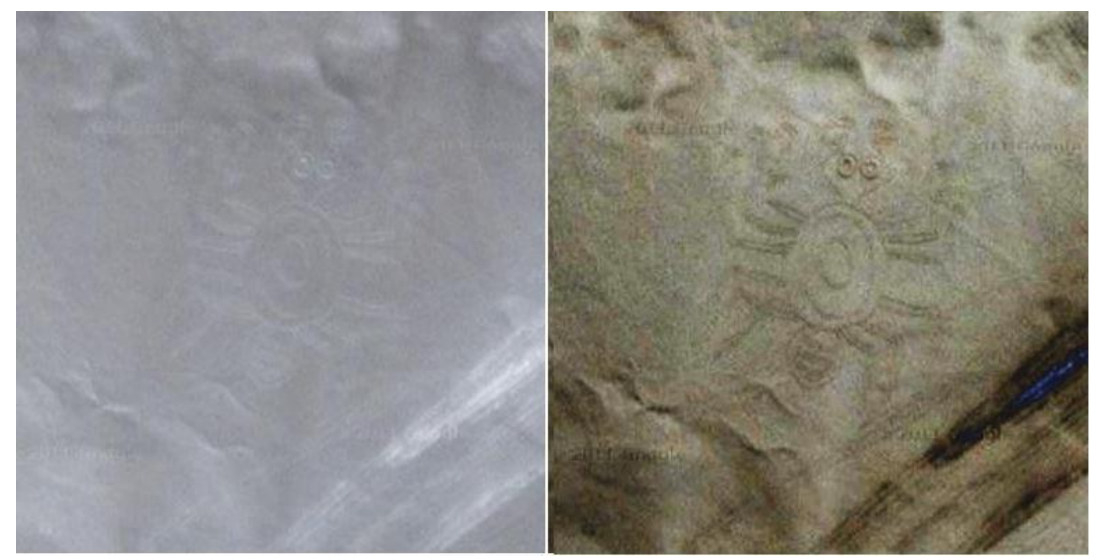

Fig. 3. Here a geoglyph in the Nazca Desert, Peru (coordinates: 14.9997S, 75.0125W). The Nazca geoglyphs, known as the Nazca Lines, are the most famous negative geoglyphs of Peru. The geoglyph was made by removing the uppermost surface, exposing the underlying ground which has a different colour. The image on the left is showing what we can

see in the Google Maps. On the right, the image after using the Gimp "Curves" tool to change the local contrast.

\section{An example on satellite images}

It is well-known that the satellite images are fundamental for several investigations, ranging from the geophysical researches [4,5] to monitoring of infrastructures [6] and historical and archaeological surveying [7]. However, these images generally need some processing [8]. Let us consider what we can have using the "Curves" tool of Gimp, for instance for an archeological investigation. The specific case could by a study with Google Earth of the geoglyphs in the Nazca Desert, Peru. The Nazca geoglyphs, known as the Nazca Lines, are the most famous negative geoglyphs of Peru [9]. Included among the UNESCO World Heritage Sites in 1994, the Lines are located in the Nazca Desert, a large region between the towns of Nazca and Palpa. The figures range in size up to several hundred meters and therefore can be better recognized from the air or in satellite imagery. On the Nazca plane, the geoglyphs were made by removing the uppermost surface, exposing the underlying ground which has a different colour. This technique produces a "negative" geoglyph.

The original images, obtained from the Google Earth or Maps (see an example in the Figure 3), of this desert surface are grey and flat, but adjusting and redistributing the contrast, the geoglyphs becomes quite visible. In the Figure 3 the "Curves" tool of GIMP had been used. The image demonstrates that this is an interesting tool for archaeological investigations. In the Figure 4, it is shown the geoglyph of a spiral as we can see it before and after processing.

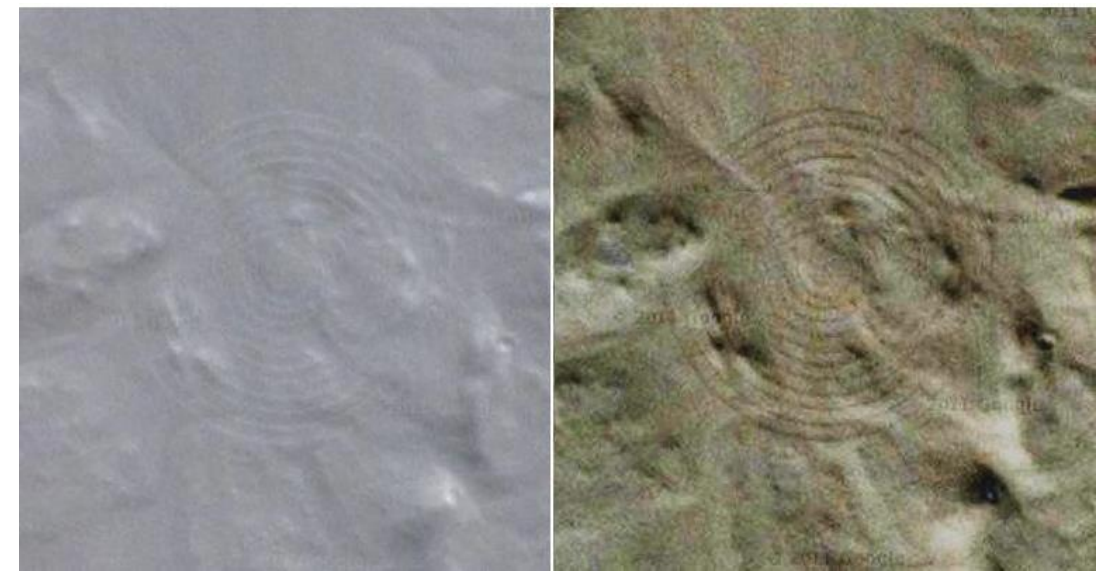

Fig. 4. Another geoglyph of the Nazca Desert, in the images before (left) and after (right) processing.

\section{Increasing the visibility}

After we have seen how some variations of images can be obtained with the curves of this tool, let us try to define a functional of these curves. That is, we consider the curves of Gimp as the analogous of the paths in the Figure 1. Let us indicate them with the index $\gamma$.

Consider a curve $\gamma$ as obtained from the panel of the tool: the brightness of the original image changes accordingly and then

$\mathrm{b}(\mathrm{x}, \mathrm{y}) \rightarrow \mathrm{b}_{\gamma}(\mathrm{x}, \mathrm{y})$

The average of this brightness is given by:

$\bar{b}_{\gamma}=\frac{\sum_{i=1}^{N_{x}} \sum_{j=1}^{N_{y}} b_{\gamma}(x, y)}{N_{x} N_{y}}$ 
where $\mathrm{N}_{\mathrm{x}}, \mathrm{N}_{\mathrm{y}}$ are the dimensions of the image. We can define the variance as a functional in the following way:

$$
V_{\gamma}=\frac{\sum_{i=1}^{N_{x}} \sum_{j=1}^{N_{y}}\left[b_{\gamma}(x, y)-\bar{b}_{\gamma}\right]}{N_{x} N_{y}}
$$

$\mathrm{V}_{\gamma}$ depends on curve $\gamma$. This is a functional because Equation 3 provides a map from curve to real number. To see the behavior of this functional as the curve $\gamma$ is varied, let us use again an image of another Nazca geoglyph. The original aerial image, quite grey ad dull is shown at the upper left corner of Figure 5. This is the geoglyph of the Condor, recoded by Raymond Ostertag [10].

The numerical value of the functional is obtained using a Fortran program which is acquiring the image manipulated with Gimp and extracting its tone map $\mathrm{b}_{\gamma}(\mathrm{x}, \mathrm{y})$. From this tone map, the program calculates $\mathrm{V}_{\gamma}$. It is the number reported in the corresponding image. Dragging the curve above or below the diagonal line simply brighten or darken the image: the output images have smaller values $\mathrm{V}_{\gamma}$. If a flexural point is created in curve, as shown in the panel at the upper right corner, $\mathrm{V}_{\gamma}$ increases with respect that of the original image. Moreover, the visibility of the image is strongly increased. The mean value (2) and variance (3) are statistical quantities, that are extensively used in parameterizing the images, for applications in several research fields. For instance, as discussed in [11], a study of textural changes in the images in liquid crystalline cells, allows to determine new phases and transitions.

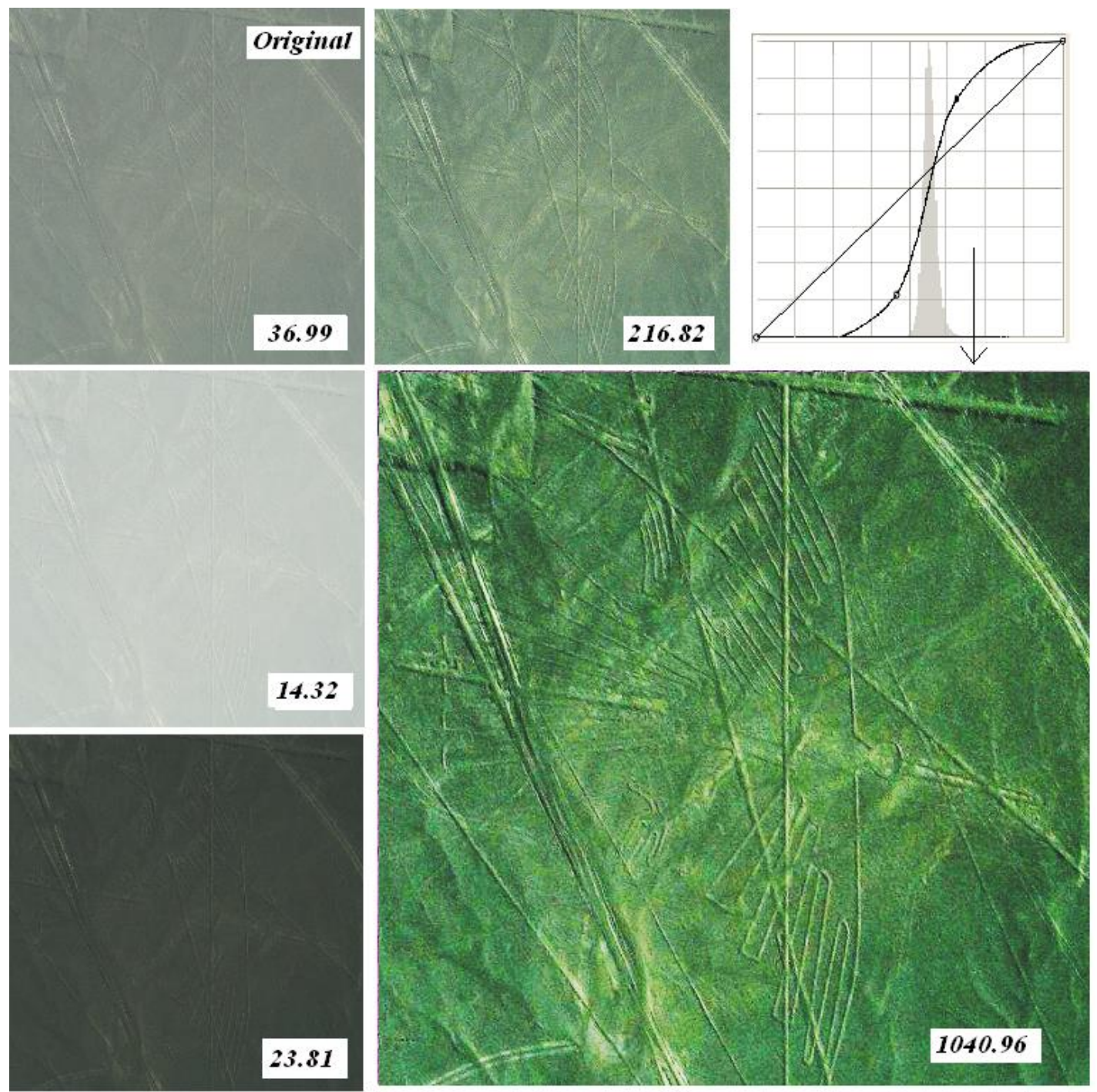

Fig.5. Let us use another Nazca geoglyph. The original aerial image, quite grey ad dull, is shown at the upper left corner. This is the geoglyph of the Condor, recorded by Raymond Ostertag. The value of the functional of Equation 3 is by means of a Fortran program; the number reported in the corresponding image. Dragging the curve above or below the diagonal line produces bright or dark images: the output images have smaller values $\mathrm{V}_{\gamma}$. If a flexural point is created

in $\gamma \square \square$ as in the panel shown at the upper right corner, $\mathrm{V}_{\gamma}$ increases with respect that of the original image. The visibility of the image is strongly increased as we can see in the panel with the green image. 


\section{To define the visibility}

As shown by Figure 5, the functional $\mathrm{V}_{\gamma}$ increases as the visibility of the geoglyph increases. Of course, a high variance $\mathrm{V}_{\gamma}$ does not mean a consequent increase of visibility: let us imagine, for instance, to change a grey-tone image in a black and white image, the variance is strongly increased but a large part of details is cancelled, and their visibility is cancelled too. To experiment this fact, we can use Gimp increasing the contrast of an image; we see that several details disappear.

We have then to find another possible functional which can be a measure of the visibility of details. Here, let us define a functional modeled on the problem of the visibility of interference fringes in optics [12]. This physical quantity is also known as the "interference visibility" or "fringe visibility". The interference of light produces a pattern of the intensities, created by bright and dark fringes. Supposing $I_{\max }$ the intensity of the bright and $I_{\min }$ that of the dark fringe, the visibility is defined as:

$V=\frac{I_{\text {max }}-I_{\text {min }}}{I_{\text {max }}+I_{\text {min }}}$

To use in the image processing this visibility, we can define the analogue of the quantities $I_{\max }, I_{\min }$ in the following manner. We consider the mean value as defined in (2), to separate the pixels in two subsets: one set contains the pixels having a brightness greater or equal that of the mean value, the other has the pixels with $b_{\gamma}$ less or equal the mean value. Each set has a number of $\mathrm{N}_{\leq}, \mathrm{N}_{\geq}$pixels, respectively. For each set, the following mean values are evaluated:

$$
\bar{b}_{\min , \gamma}=\frac{1}{N_{\leq}}\left[\sum_{i=1}^{N_{x}} \sum_{j=1}^{N_{y}} b_{\gamma}(x, y)\right]_{b_{\gamma} \leq \bar{b}_{\gamma}} \quad ; \quad \bar{b}_{\max , \gamma}=\frac{1}{N_{\geq}}\left[\sum_{i=1}^{N_{x}} \sum_{j=1}^{N_{y}} b_{\gamma}(x, y)\right]_{b_{\gamma} \geq \bar{b}_{\gamma}}
$$

Therefore, the analogous to the intensities are two mean values of the two subsets of the image pixels. In (5), the dependence on $\gamma$ is explicitly written. Let us define the visibility, analogous to the fringe visibility, as:

$$
V_{\gamma}=\frac{\bar{b}_{\max , \gamma}-\bar{b}_{\min , \gamma}}{\bar{b}_{\max , \gamma}+\bar{b}_{\min , \gamma}}
$$

Assuming $\mathrm{V}_{\gamma}$ as a measure of the image contrast, and therefore of its visibility, we could try to arrange some calculations in order to maximize this functionals. We could for instance assume a theoretical variation of the brightness of the grey tones in the following manner:

$$
\begin{array}{ll}
b_{\gamma}=b+a_{1}|(b-0) \cdot(b-\bar{b})|^{\alpha} & b \geq \bar{b} \\
b_{\gamma}=b+a_{2}|(b-255) \cdot(b-\bar{b})|^{\beta} & b \geq \bar{b}
\end{array}
$$

where $b$ are the brightness of the original image, and find the parameters $a_{1}, a_{2}, \alpha$ and $\beta$ which are

maximizing (6). These four parameters can span some suitable ranges. In the numerical calculation on the image map by means of a Fortran program, we avoid those parameters giving $b_{\gamma}$ greater 255 or lower 0 . Parameters $\alpha$ and $\beta$ range from 0.1 to $1.0 ; a_{1}$ from 0 to 5 and $\mathrm{a}_{2}$ from 0 to 3 . After maximizing (6), the Fortran program gives us the output image.

Let us see how the variations (7) and the maximization of (6) are working on the image of the Nazca geoglyph used for Figure 5. Using a grey-tone version, we can have the image in the Figure 6 . The parameters of the variation giving the maximum value of visibility (6) are $\alpha=0.3, \beta=0.5, \mathrm{a}_{1}=4.5$ and $\mathrm{a}_{2}=1.2$; this result is limited to the variations having brightness in the range $[0,255]$. Note that, with respect to the original image (see the Figure 5 and [10]), the visibility of the details is strongly increased. 


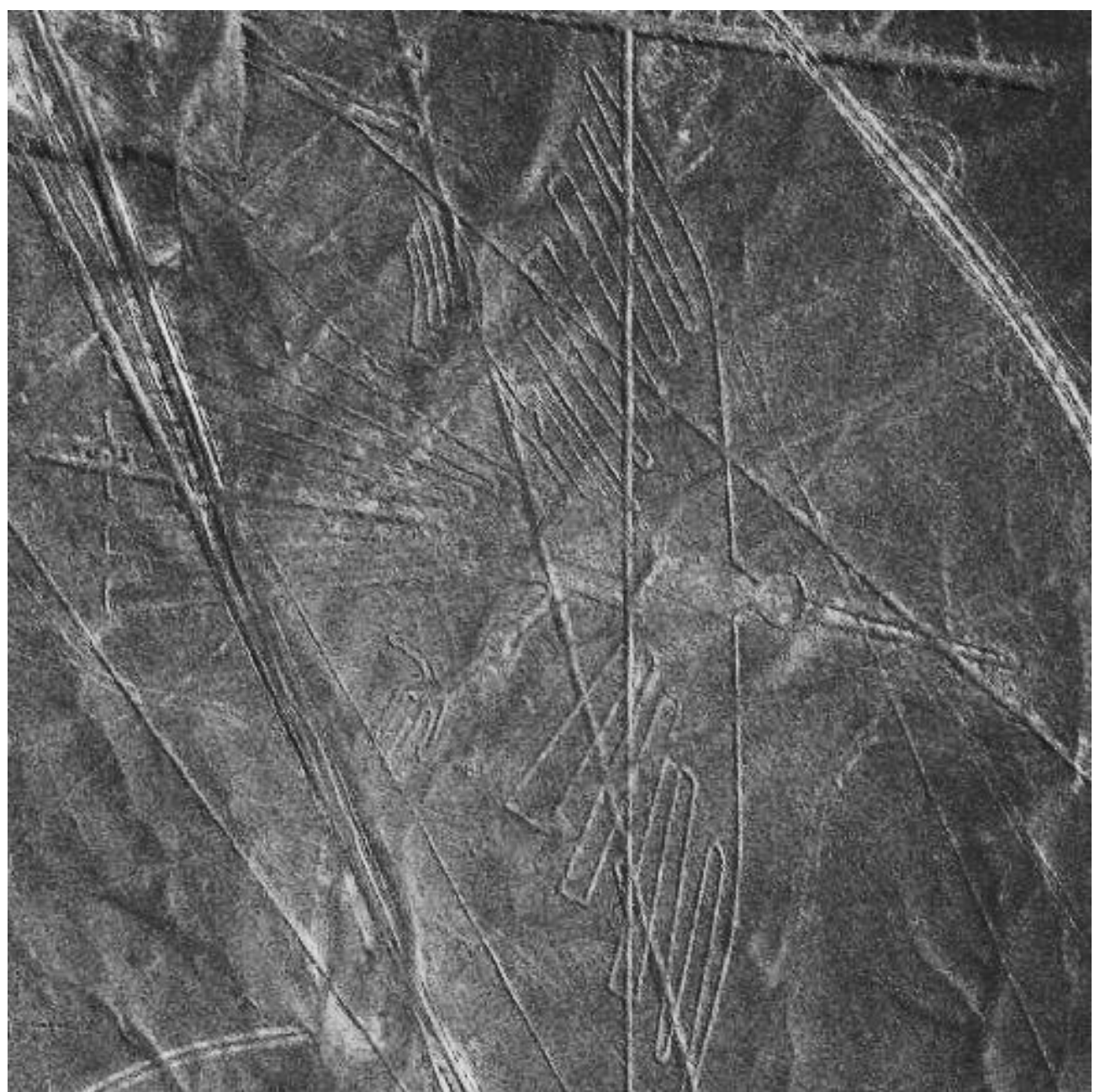

Fig.6. This is the image of the Nazca geoglyph which is maximizing the visibility given by Eq.6. Compared to the original image, the visibility of details is strongly increased.

\section{Conclusion}

Of course, other functions instead of (7) can be devised and used to create the image variations, and other functionals can be proposed to measure the image visibility. Here, the aim of the paper was that of illustrating the calculus of variations with an experimental approach. For this approach, I used a tool of Gimp, in order to visualize how the image variations can be. After the discussion of this tool, which is able to strongly increase the visibility of images (quite interesting for archaeological survey), I have discussed a visibility functional analogous to the fringe visibility of optical interference. This approach seems to give good results.

\section{References}

1. G. Aubert, P. Kornprobst, Mathematical problems in image processing. Partial differential equations and the calculus of variations. Applied Mathematical Sciences, n.147, New York, 2006, Springer Verlag.

2. R.P. Feynman, R.B. Leighton and M. Sands, The Feynman Lectures on Physics, 1963, California Institute of Technology, Addison-Wesley.
3. GIMP, GNU Image Manipulation Program, http://www.gimp.org/ 4. S.J. Whitmeyer, Google Earth and Virtual Visualizations in Geoscience Education and Research, Geological Society of America, $01 /$ gen/2012.

5. A.M. Scheffers, S.R. Scheffers and D.H. Kelletat, The Coastlines of the World with Google Earth: Understanding our Environment, Springer, 06/mar/2012.

6. R. Grayson and Chimed-Erdene Baatar, Remote sensing of crossborder routes between Mongolia and China, World Placer Jounrla, volume 9, 2009, pages 48-118.

7. A.C. Sparavigna, The satellite archaeological survey of Egypt, Archaeogate, Egittologia, 6 June 2011.

8. A.C. Sparavigna, Image Processing for the Enhancement of Satellite Imagery, In: Image Processing: Methods, Applications and Challenges, Vítor Hugo Carvalho, Nova Science Publishers, Inc. (USA), 2012, pages 149-161.

9. A.C. Sparavigna, Lines, dots and spirals on Peruvian land, Archaeogate, Antichità Classiche, 16 September 2011.

$10 . \quad$ Raymond Ostertag, http://en.wikipedia.org/wiki/File:Nasca_Condor_2007_08.JPG

11. A. Sparavigna, A. Mello, B. Montrucchio, Texture transitions in the liquid crystalline alkyloxybenzoic acid 6OBAC, Phase Transitions, volume 79, 2006, pages 293-303. For references on the use of statistical methods in image processing, see please the references therein.

12. M. Born, E. Wolf, Principles of optics: Electromagnetic theory of propagation, interference and diffraction of light, 1997, Cambridge University Press. 